\title{
Antimicrobial stewardship in central eastern european countries
}

\author{
Rudolf Anschober
}

The use of antimicrobials is a central element in modern medicine. However, over the past decades the threat of antimicrobial resistance has steadily increased and has caused a high level of international concern. It is necessary to take urgent measures against the growth of antimicrobial resistance because a broad field of modern medical therapy will fail without effective antimicrobials.

In the past, new antimicrobials with new mechanisms of action entered the market regularly leading to a constant availability for all types of diseases. Nowadays only very few new antibiotics are developed, which means that those already existing should be prescribed with caution. Otherwise, there may be a future without potent antimicrobials.

An important measure to prevent the further growth of antimicrobial resistance is the rational use of existing antimicrobials. One fundamental measure to reach this target is antimicrobial stewardship. This means implementing a correct diagnostic approach and subsequently, the use of the appropriate antimicrobial. Furthermore, this includes prescribing the right dosage form as well as an adequate duration of therapy and a switch of dosage form as applicable. In many cases, antimicrobial stewardship does not only improve the therapy for the patients but also leads to a reduction in the use of antimicrobials and a decline in treatment expenses.

Antibiotic resistance is a global challenge that requires global action. The WHO has identified antimicrobial resistance as one of the most critical health threats for the years to come and strongly recommends implementing national action plans. The exchange of best practice models in the field of antimi-

Federal Minister R. Anschober $(\bowtie)$

Federal Ministry of Social Affairs, Health, Care and

Consumer Protection, Stubenring 1, 1010 Vienna, Austria rudolf.anschober@sozialministerium.at crobial stewardship as provides by this publication is extremely important in this constant fight for saving our effective antimicrobials.

Austria plays a central role in this area and it is our intention to cooperate closely with our neighbours in the CEE region. Therefore, the Austrian Ministry of Health is very happy to support this initiative of the Semmelweis Foundation in order to foster the cooperation between all CEE countries by presenting and evaluating their national antimicrobial stewardship plans in a supplement journal of the Wiener Medizinische Wochenschrift. We will only win the battle against resistant microbes, if we work together across borders, by learning from each other and by adapting systems that have proven to be successful elsewhere.

With this publication, the Semmelweis Foundation pays tribute to the Austro-Hungarian physician Prof. Dr. Ignaz Semmelweis, who has laid the basis for hand-disinfection and infection prevention.

Conflict of interest R. Anschober declares that he has no competing interests.

Publisher's Note Springer Nature remains neutral with regard to jurisdictional claims in published maps and institutional affiliations.

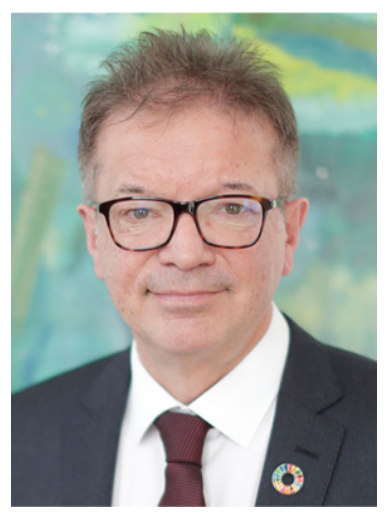

Rudolf Anschober

(c) BKA / Andy Wenze 